\title{
Color Sensation in Figurative Painting
}

\author{
Alberto Carroggio De Molina
}

Department of Drawing, University of Barcelona

*Corresponding Author: alcarroggiode@ub.edu

Copyright $\odot 2014$ Horizon Research Publishing All rights reserved.

\begin{abstract}
The figurative painter accesses very complex levels of knowledge. To produce a painting requires, first, a deep analysis of the image of the reality and, afterwards, the study of the reconstruction of this reality. This is not about a process of copying, but a process of the comprehension of the concepts that appear in the representation. The drawing guides us in the process of the production of the surface and in the distribution of the colours that, after all, are the data with which the vision mechanism builds the visual reality. Knowing the colour and its behaviour have always been a requirement for the figurative painter. From that knowledge we can draw wider conclusions.
\end{abstract}

Keywords Perception Color Drawing Painting, Relativity

\section{Introduction}

Painters never have been too explicit and our pronouncements have been scarce and almost always oriented to give practical advices about our profession.

But the figurative painter accesses very complex levels of knowledge. To produce a painting requires, first, a deep analysis of the image of the reality and, afterwards, the study of the reconstruction of this reality. This is not about a process of copying, but a process of the comprehension of the concepts that appear in the representation. The drawing guides us in the process of the production of the surface and in the distribution of the colours that, after all, are the data with which the vision mechanism builds the visual reality. Knowing the colour and its behaviour have always been a requirement for the figurative painter. From that knowledge we can draw wider conclusions.

\section{The Sensation of Colour}

The sense of colour is the result of a process which starts in the eye and finishes in the occipital lobe of the brain. This process, which allows us to see all that surrounds us, is the corresponding energy that normally is electromagnetic. Nonetheless, if we close our eyes and press the eyeball, we see little stars or blobs of colour. That is, we contribute sufficient energy to the organ of vision to unleash the visual process.

What is the difference between the vision that we normally have about our environment and the one which is produced as a consequence of pressing the eyeball? We will have two different images: one of them, the one of our environment, is an image formed by the organized distribution of the visual data, and the other, which is the consequence of pressing the eyeball, is a disorganized distribution of the visual information from which we won't draw any conclusion.

We would say that the image that we obtain as a consequence of pressing the eyeball is an internal vision, that is to say, it is produced inside our body, and the other one is external, in other words, it is produced outside it. Now, the image of our environment is composed of colours. If we look at a photograph or a painting we will see that we can only find blotches of colour in them, that is to say that the things that are represented therein are only formed by colours. We know that colour is formed in the brain, therefore, if images are compound of colours and colours are formed in the brain, thus we must conclude that if the images are composed of colors and the colors are formed in the brain, we must conclude that both are internal images and, as a consequence, our vision of the world is - lacking a better expression- an "autistic" process. The image that we have of things is a product of our mind, because colours don't exist outside it. Consequently, what we consider our visual external environment is just a phenomenon generated inside our brain and we can be sure that it will not go out of it.

Naturally, the rest of the senses behaves in the same way because they are localized in the same space we have created for vision. They are integrated in a single field since the things we see are the same as those we touch, smell, feel and hear. So it is not only vision that behaves as an autistic mechanism, but rather all the sensorial information processes in the same way.

We can deduce, then, that the elaboration of reality in any of its levels is not a photographic process. There is no "real" reality, a reality which is platonic, alien to us and with a previous existence of which we obtain a copy, but rather any form of reality of which we are conscious has been elaborated by our mind. 
Man, then, cannot consider his exterior as something different from himself. The self is formed by all the conscious reality that the mind is capable of creating. This includes both the body and the entire universe that surrounds it. The self is not a physical being isolated from its environment, nor is it a supposed immaterial entity, that deep self we call spirit, but rather it is composed of whatever the mind is capable of creating.

We do not have access to any form of consciousness before it is elaborated by the corresponding mental mechanism. Any form of consciousness comes determined by the mental mechanisms that generate it. And although we can suppose that something must exist at the edge of our perception, we will never be able to know how much we know about its real nature.

A sensation cannot be called "sensation" until it reaches consciousness. We cannot determine the existence of a colour until we can be conscious of it. We can investigate physiologically the formation of the sensation or find out in which zone of the brain it is elaborated, but we cannot be conscious of a sensation outside our consciousness, since it is not possible to be conscious of someone else's consciousness.

My consciousness - which is the only one that exists - is only conscious of two things: sensations and concepts ideas. Sensations have a determined characteristic: we cannot describe them. Sensation is a discrete act of consciousness. Let us see, as a example, what happens with colours. We can describe colour as an abstract element, but colour as a sensation is indefinable and inexplicable. What is the colour red? How would we explain to a person born blind the sensation of red? We would feel the same powerlessness if we had to explain sound to a deaf person.

We know that a definition explains the elements that form the whole of what we intend to describe. And this demands that the component elements exist before the whole of the thing. If not, it would be possible that the whole would exist without one of its parts. When something cannot be described, it means either that it doesn't exist or that its existence doesn't depend on its component elements, but rather that it emerges directly from consciousness. The latter is what happens with sensations. We cannot define red, or any other colour, because nothing exists at the level of consciousness previous to it. The essential difference between sensation and concept is that a concept can be defined while sensations cannot.

In the process of constitution of concepts, there is an important issue: the qualitative step from sensation to something which we can designate as "simple concept". By way of illustration: a surface is a simple concept since it is the "sum" of sensations, which are the result of a "sum" of concepts. I think that the act of addition, or perhaps we must call it integration, depends on an innate faculty and the step from sensation to concept depends on this capacity. A sensation, by itself, does not offer more data than its own self. In other words, the assistance of some mechanism that integrates the sensations in more complex products is necessary. In this way, the visual data - colour stains- are organized in visual surfaces.

Therefore we need this integrating mechanism to define a simple concept since it depends on two factors that existed previously: the sensation and the faculty of integration.

We will define, then, a surface as the sum of organized and discrete sensations, and this definition can be applied to both the tactile concept and the visual concept of surface.

It is true that we have created a mental mechanism capable of integration, whether it be this or a similar one. This mechanism must exist since we, as painters, have used it to create tridimensional visual elements using bidimensional surfaces. And we use this mechanism in both senses: we can disintegrate the surface into colours and, afterwards, restore it again. If we observe an object and we break it into colour stains, we can see that, at the beginning, we can keep the vision of the object, but if we carry on the process, at a certain point we will perceive it so separately that the object will disappear - not only the vision of the object, but the vision of its surface - any surface. In other words, we will invert the process of formation of the surface. When, over a bidimensional surface, the painter distributes the colours and creates visual tridimensional forms (which, at a tactile level is still bidimensional), he is manipulating the elements that forms the image to build surfaces at his own whim. So much so that the painter can "see" whatever surfaces he wants and orient it in the space as he wants. He can also visually convert the originally bidimensional surface into a tridimensional one. That is to say, he sees in the painting the surface of the object that he wants to represent that, deep down, can be anything.

So we can consider that the concept of surface is universal. On a visual level, surfaces of different natures do not exist: the surface of the canvas, visually, can be converted into a hand, a loop or a bracelet. The only category of the visual surface is form. Because not even colour can be considered as an attribute of surface. With colour we can create surfaces, but surfaces of only one colour do not exist, because to build a visual surface it is necessary to integrate different colours. This is the discovery of Velázquez: the reality is composed of many discrete colour stains and, if they fit together correctly, we can create automatically the surface of the object we want to represent. In the illustration of fig. 1, the viewer can choose to see the objects as such, or to perceive the blotches that form them. It is easy to observe the colours that configure the red loop and see them as isolated stains, but, when colours are perceived separately, we lose the complete vision and the object.

This fact warns us how the mind creates its environment unconsciously since, given an order of colours, the corresponding surface is automatically generated.We can, then, consider this integrational ability as an innate mechanism capable of organizing sensations and elaborating new forms of consciousness without manifest intervention of our will. Each sensorial mechanism follows its own procedure and goes by its own rules. In vision, the answer is relative because it is not directly proportional to the 
stimulus. This is in contrast to sound;a Do always sounds as such, whether it sounds before or after a Re o a Mi. On the other hand, the amount of sound has a relative behaviour: if we come out of an extremely noisy locale, the noise of the street, which seemed to exceptionally loud to us before, will seem agreeable now.

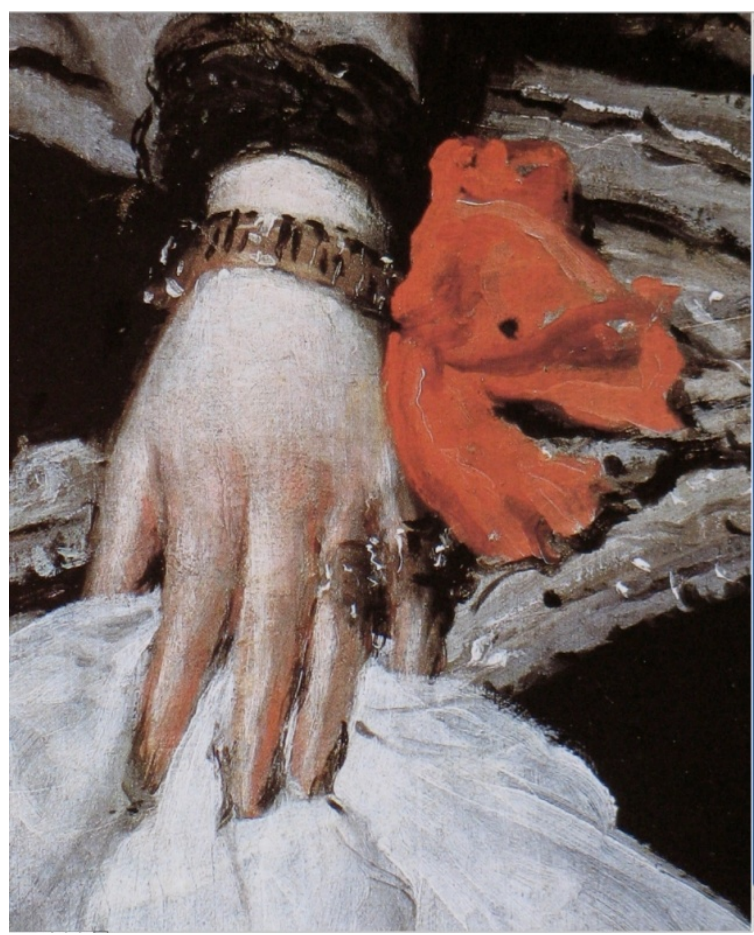

Figure 1. Velázquez, "Mariana de Austria”, Museo del Prado, (fragment)

With colour, over different fields, the same stimulus generates different responses. That is to say that the same pigment can be seen in different colours. The two frames of fig. 2 have been done with the same pigment of the colour's RGB selector: red 100 , green 60 , blue 20 , of the computer program "GraphicConverter". However, their colour is different and will vary again over any other background. It is the same with the greys range of the aforementioned software.
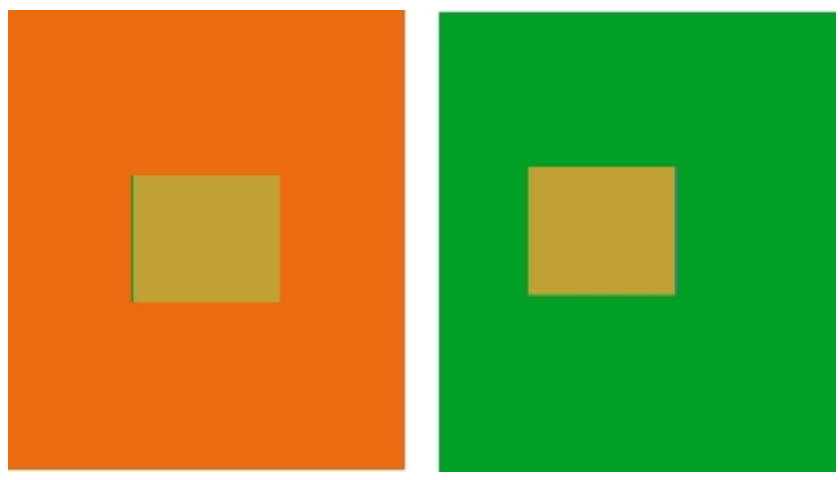

Figure 2. A. Carroggio, drawing with computer

The same thing happens on the grey scale of fig. 3; the interior "frames" are the same grey from the grey scale of the aforementioned program.
We cannot assume that there exists a perfect background in which the colour produced in its interior would be the "correct" colour. Therefore, we would wonder: which is the authentic colour? Which, among the two interior colours that we can see in the sample illustrations, are the colour that we should see? We must conclude that colour is not the pigment, nor the amount or quality of the energy that trigger the visual process. Colour is what we see or, in other words, the response of the mechanism. This response depends both on the visual field and on the particular conditions in which the visual phenomenon is produced. Among these conditions we can even find our state of mind, which will have an effect on the response of the mechanism.
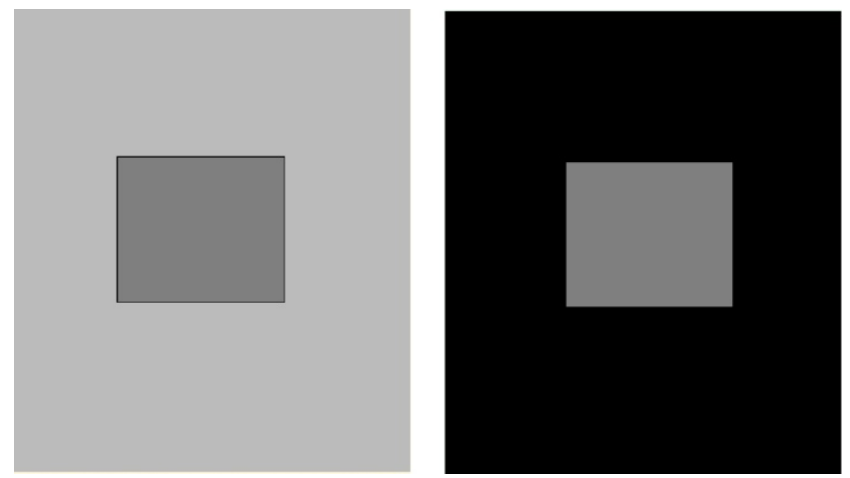

Figure. 3 A. Carroggio, drawing with computer

We can measure, then, the intensity of light, its frequency or its temperature. We also can analyze the composition of pigments. However, the response -colour- is only measurable by the individual that perceives it as a pure act of consciousness.

We must conclude that reality, in any of its aspects, depends on the mental mechanisms by which is created, and its characteristics are determined by the categories of these mechanisms. We will exemplify it by following these principles:

We know the difficulties that we find when we try to define "time". If it were a concept we would be able to define it. That would mean that it is formed by elements with a previous existence. In other words, something that had existed before time, which would be contradictory. And since we said that our brain is only conscious of two products - sensations and concepts- we must consider it as a sensation and, in fact, it behaves like a sensation. Our perception of time is relative because, like colour, it depends on the mental mechanism that creates it. Basically, it depends, as we all know, on our state of mind. Watches give a conventional measure of time, but that does not mean that they can measure the real value of time. To aspire to an absolute measure of time is the same as trying to obtain an absolute measure of colour.

\section{Conclusions}

Since, as well as color, time is just a sensation and it does 
not exist outside our minds, it would be absurd to assign measurable values to it, same way as it would be illogical to apply values to color. Therefore, we cannot either consider velocity and space as measurable parameters outside our conscience.

Scientists and artists, as spectators of a phenomenon -any phenomenon- achieve, ultimately, the same product. After all, their ways to understand their environment must converge because its mental mechanisms are the same.

The figurative painter knows he cannot try to represent objects, since objects are indefinable in a physical reality level. He must accept that the only way is to represent a completely subjective image. Solipsism is a normal state for the figurative painter, as he knows that the image he sees is exclusively made up of colors - the colors which he created and that his only function is know how to distribute those colors in a canvas. This is actually the only thing painters can do, since they do not have any other tools.

This approach may compel us to reconsider our knowledge about the Universe and can make us differentiate between the known universe - the interior universe- and the exterior universe, and find out which knowledge belongs to one or another.

The truth is solipsism is nowadays philosophically discredited, and is a very uncomfortable trend, specially for science. Nevertheless, the former exposition demonstrates that, at a physiological level, we need to change the vision of our field of knowledge. We have to ask ourselves: ¿How sure we can be about whether the equations of the scientists describe Universe's behavior or are just a display of their intellect's capacities?

\section{REFERENCES}

[1] ALBERS, J."La Interacción del color". Alianza. Madrid, 1984.

[2] CARROGGIO, A."Estética en la pintura: el hecho de pintar" $1987 \mathrm{http}: / /$ hdl.handle.net/2445/36692

[3] CARROGgIO, A."La relatividad en la pintura figurativa" http://hdl.handle.net/2445/13542, 2010

[4] COHEN, J."Sensación y percepción visual". Trillas. Méjico, 1979.

[5] GROOM, E. “Sensaciones Visuales", Universidad Central de Venezuela, Caracas, 1972

[6] HUYGHE, R."Los poderes de la imagen". Labor. Barcelona, 1971.

[7] ORTEGA Y GASSET, J."Unas lecciones de metafísica", Alianza Editorial, Madrid, 1966.

[8] VERALDI, G."Psicología de la creación". Bilbao, 1974.

[9] VILLAFAÑE, J."Principios de la teoría de la imagen". Pirámide. Madrid, 1996. 